\title{
Computer Vision Based Industrial Robotic Arm for Sorting Objects by Color and Height
}

\author{
Abu Salman Shaikat*, Suraiya Akter, Umme Salma \\ Department of Mechatronics Engineering, World University of Bangladesh, Dhaka - 1205, Bangladesh
}

Received: October 1, 2020, Revised: December 02, 2020, Accepted: December 04, 2020, Available Online: December 09, 2020

\begin{abstract}
In industrial production systems, manufacturers often face difficulties in sorting different types of objects. Color and height-based sorting which is done manually by human is quite a tedious task and its needs countless time as well. For manual sorting, many workers are required, which can be quite expensive. Moreover, robots that can sort only color or height can't be effective when there is a need of products with same color with different heights and vice versa. In this paper, a computer vision based robotic sorter is proposed, which is capable of detecting and sorting objects by their colors and heights at the same time. This work isn't done before as height sorting of same shapes is a new technique, which is done with color sorting techniques by computer vision. It is equipped with a robotic arm having 6 degree of freedom (DOF), by which it picks up and then place objects according to its color and height, to a predetermined place as per the production system requirement. A camera with the computer vision software detects various colors and heights. Haar Cascade algorithm has been used to sort the products. This multi-DOF robotic sorter can be a remarkably useful tool for automating the production process completely, where multiple conveyor belts are used, which can reduce time complexity as well. In the proposed system, the efficiency of color and height sorting is around $99 \%$, which proves the efficiency of our system. The overall improvement in the efficiency of the production process can be significantly enhanced by using this system.
\end{abstract}

Keywords: Color and Height Detection, Sorting, Computer Vision, Degree of Freedom, Robotic Arm, Efficiency.

This work is licensed under a Creative Commons Attribution-Non Commercial 4.0 International

\section{Introduction}

For over two decades, industrial automation has transformed itself into a more adaptable and programmable automation from its earlier fixed self with the recent technological advancement. The combined field of electrical, electronics, mechanical, and computer vision-based control system has substituted the manual workers, i.e. human beings. Intelligent machines have enhanced both productivity as well as comfort. Usually, industrial robot is used to carry out the monotonous and recurring pick and place tasks; for example, placing the parts from and to a conveyor belt, managing Computerized Numerical Control (CNC) machineries, stowing the parts to and offloading these from the machines. It is extensively used in the industrial assembly line. Industrial robot has proven its worth in this regard within the past few decades. In addition, it sets the tempo of the operations up for boosting the production rate. Robots have been used for quite a long time to automate the flexible product manufacturing system. Robots are turning into more than just machines by using Artificial Intelligence (AI), thus are in great demand in recent years. Robots have solved the expensive labor issues. They also satisfy and fulfill rapidly increasing demands from the customers. Although the expense of assembling a robotic system has relatively increased, performing the intricate, hazardous and insecure tasks competitively has made the robots preferable than their human competitors. Robotic automation not only enhances the production capability, but also offers efficient and improved solutions in numerous scenarios.

In this paper, an autonomous robotic arm is proposed, which can identify objects from the conveyor belt and transfer these to the desired location based on their colors. In an industry dealing with a large number of products, this autonomous robotic sorter can be used in the production system for keeping the expenses below the margin. The robot has been designed stationary around its platform. It uses camera to collect information regarding the colors and heights of the objects. The robot has the capability of identifying objects' colors and heights by placing those in front of its camera as well as sorting out based on color. After sorting, it relocates objects to the destined location.

OpenCV and .NET framework are used as software tools to sort the products in accordance with color and height. Haar Cascade algorithm has been used for Computer Vision (CV) methods. Furthermore, Arduino Nano has been employed for moving or picking the products from specific areas and placing into different locations taking help from $\mathrm{CV}$ method.

\section{Literature Review}

In recent years, researchers and engineers have developed and assembled various types of pick and place robots for carrying out industrial operation. Among them, Dhayalini et al. 0 presented a method for separating deterioration \& nondeterioration waste by utilizing a pick and place robot. A simple $\mathrm{CV}$ technique for robotics had been implemented in their work which is Android mobile phones based. It solved the issue of separating degradable and non-degradable wastes in streets, which are separated by human beings with a risk of facing communicable diseases. Android mobile had been used to detect the degradable, i.e. vegetables, and non-degradable, i.e. plastic cover objects and then send that retrieved information to the connected microcontroller unit through a Bluetooth module. After receiving that desired information, microcontroller unit operated the robotic arm for reaching the particular objects. Receiving ' 1 ' from the Bluetooth indicated the non-degradable object and receiving ' 0 ' signified the degradable objects. The 
robotic arm acted accordingly and took the separate objects for putting those into the separate boxes.

$\mathrm{Hu}$ et al. 0 developed a vision-based Selective Compliance Assembly Robot Arm (SCARA) system for obtaining spanners from the targeted location and putting these to desired location. The system was designed to be highly appropriate for the traditional factories. All the component materials are constructed from plastic and aluminum, apart from the motors. The SCARA had around $50 \mathrm{~cm}$ of ranges for working. It was equipped with 4 degree of freedom (DOF). The vision system process can be divided into three major sections, such as calibrating image, recognizing image and finally, localizing objects. Firstly, reforming the image distortion had taken place in image calibrations step. Secondly, the spanner located in its workplace had been captured as image and segmented by applying information regarding color in the step of image recognition. Finally, the information about the spanners was retrieved, for example, the center, end to end length, incline and length related tool number. Modbus-TCP communication interface was used to enable secure transmission of position and rotation command to motion controller.

Gecks et al. 0 built an industrial robot system, where a number of stationary cameras were assembled to oversee the workspace for safeguarding human-robot teamwork. Every robot transfer motion was scrutinized to avoid collision using obstacle detection through several CV approaches. The motion path of a robot was altered every time the possibility of a collision had been perceived. The algorithm, that had been proposed, assisted robots in executing secure pick-and-place operations by showing real time responsive activities.

Kato et al. 0 shared the idea of flexible robot manipulators which is suitable for construction with inexpensive setting. The flexible robotic arm had been developed to carry out pick and place tasks involving lightweight manufactured goods. The robotic arm was set in motion through a couple of DC motors along with a stepping motor. To control the twisting mechanism and to twist the flexible link, a wire was attached to the stepping motor. The wire was pulled to reduce the intended vibration of the flexible link's end tip.

Andhare et al. 0 transformed and mapped the 2D coordinates of an object by transforming pixel coordinate to real world coordinate. They integrated robotic arm controller on the basis of object location and orientation. They successfully picked and placed objects by placing under the vision sensor.

Li et al. 0 illustrated a new elliptical trajectory planning algorithm as the increasing speed of industrial pick and place robots requires optimization of the kinematic characteristics to operate high speed application efficiently. Their proposed algorithm was able to enhance the performance to operate the pick and place tasks. One elliptical trajectory defined with a precise secured height was created. In addition, one customized sine wave movement profile was commenced into that trajectory. The simulation results exhibit the efficacy and appropriateness of the process for implementing the industrial applications.

Lin et al. 0 assembled a robot vision system for detecting diverse target objects as well as their poses, which can be integrated in robot programming by demonstration, i.e. robot $\mathrm{PbD}$ in short. Lately, robot $\mathrm{PbD}$ has stepped into the trending topic scenario regarding robotics field. Although programming the robots is a prolonged procedure, needing deep technical knowledge, robot task programming can be streamlined by $\mathrm{CV}$ system. By analyzing human demonstration, this was implemented. They took the pick-and-place activities as robot $\mathrm{PbD}$ example. Several non-overlapped objects were positioned on a table and people picked one of those objects and moved to other place, one at a time. The robot could refine its next decision by acquiring initial information prior to learning from the human demonstration. For example, if the robot had detected the intended object and attuned its stance afterwards, then it was able to operate smoothly. Choosing the kind of gripper and/or the angle of grip beforehand helped the robot enhancing its operation. The developed robot vision system showed improvement concerning reciprocal trainability. The graphical user interface (GUI) helped in this regard. Common users, i.e. not experts, could easily choose the understandable features using the user friendly GUI. An uncomplicated scale-invariant pose assessment method was also projected. The system demonstrated itself as a feasible and effective one, sustained by the experimental results.

Lukač et al. 0 presented program code to control industrial robot. Version 2.2.2 of the renowned simulation software KUKA Sim Pro was used to run the robot cell simulation. The components, that were used, had been brought mostly from the incorporated library. However, some components were partially assembled from the scratch. The industrial robot, namely, KUKA KR6 R900 sixx, is considered to be one of the fastest robots in the current world.

Rahman et al. 0 developed a robotic sorter competent for identifying and sorting objects with different colors. It comprised of a robotic arm that picked up objects and placed those to a preprogrammed location after detecting colors. A Pixy cam supported by the CV software identified several colors. For robotic arm rotation few servomotors were used-09. The arms had the ability to move both horizontally as well as vertically concerning its base. Roshni et al. 0 proposed another technique for distinguishing objects from their surroundings by employing $\mathrm{CV}$ approaches and calculating their center of gravity.

Some researchers work with color and size detection individually, whereas very few researchers work with color and size detection together. They work with individual shape (triangular, rectangular, circular) detection only with different colors. However, in our work, we work with same shape (rectangular) with different height materials including color sorting techniques, which makes our work unique. This work can be very useful when there will be a need of sorting diverse products by its colors and just changes of heights.

The aforementioned works have some limitations. While most of these works have focused on the task of detecting and picking up objects, load calculation has been disregarded to some extent. Moreover, the force of motors should be measured for maximum efficacy. In this work, a CV based intelligent color and height sorting pick and place robot using Haar Cascade method is developed which can sort diverse products for assorted purpose. The maximum load of the robot manipulator arm is calculated that can be carried or supported by the arm. Also, the force of gripper motor and all other joint motors is computed in this work.

\section{System Design}

In order to assemble the system, some aspects were taken into consideration:

i. Servo motors are used due to its motion freedom and also, low power requirements and light weight make it suitable for designing in robotic arm. 
ii. The power is supplied from a rechargeable nickel metal hydride (Ni-MH) battery.

iii. A continuous path controller, i.e. Arduino Nano was chosen and employed. Forward Kinematics is used to control the movement of robot in every direction and position from end effector to joint parameters. Force feedback is done with the help of kinematics. Error sensing feedback control is done by servo motors.

Table 1 shows the details of robotic arm.

Table 1 Details of Robotic Arm

\begin{tabular}{llll}
\hline Motor Axis & Part & Motion & Motor \\
\hline A-Z & Base & Rotation & Servo \\
B-Y & Shoulder & Up-Down & Servo \\
C-X & Elbow & $\begin{array}{l}\text { Forward- } \\
\text { Backward }\end{array}$ & Servo \\
& & & \\
B-Y & Wrist & Up-Down & Servo \\
E-Y & Gripper & Up-Down & Servo \\
F-Linear & Gripper & Open/Close & DC \\
\hline
\end{tabular}

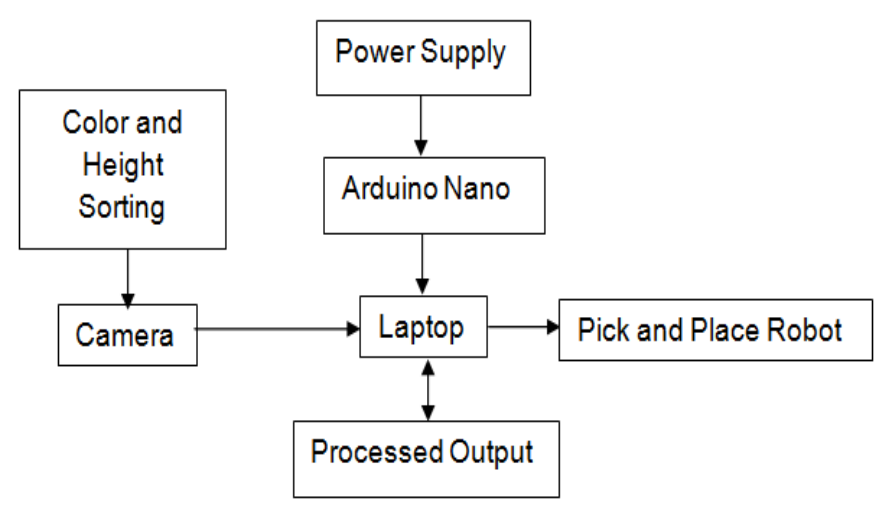

Fig. 1 Block Diagram

The prototype system has been developed using Haar Cascade method 0. For color detection, we use the function cv.cvtColor() and extract the color from the object. For height detection, Haar features have been used in CV approaches to categorize the pixel intensity by tracing inside a region. Haar features are symbolized as the rectangle regions in the image. We used edge detection technique for height detection, whereas the upper part is white, and lower part is black. For small object detection, two third of the object is white and one-third is white, whereas for black, it's just opposite. In this system, background is preferred as white for detecting the white parts without any contradictions. Object detection module trained the 300 different object pictures (30 pictures for each object) as positive images. As negative images, detection module is trained 180 pictures that should be outside of object like still picture, background and people's face. The classifiers comprise of couple of or three rectangle features for scanning features incessantly in the window. Since this scanning is a tedious and repetitive task, Cascade classifier is introduced to shed the overwork. It arranges a set of features with positive and negative images into an assorted level of classification structure 0 . It defines an upper and lower threshold value for both the object sizes. One detection per object is introduced, which reduces overlapping detection, which can increase the performance of the system. The block diagram is depicted in Fig. 1. First of all, the camera is placed in standby position and the OpenCV window is opened afterwards. Then, the camera is activated in OpenCV and the objects are sorted as per color and height. The result is revealed in OpenCV. Finally, system is run to start the pick and place robotic movement.

Fig. 2 represents the arm of the robotic manipulator. All the diameters from link to link are shown here. Mechanical design of the pick and place robot is shown in Fig. 3, which includes all except the object.

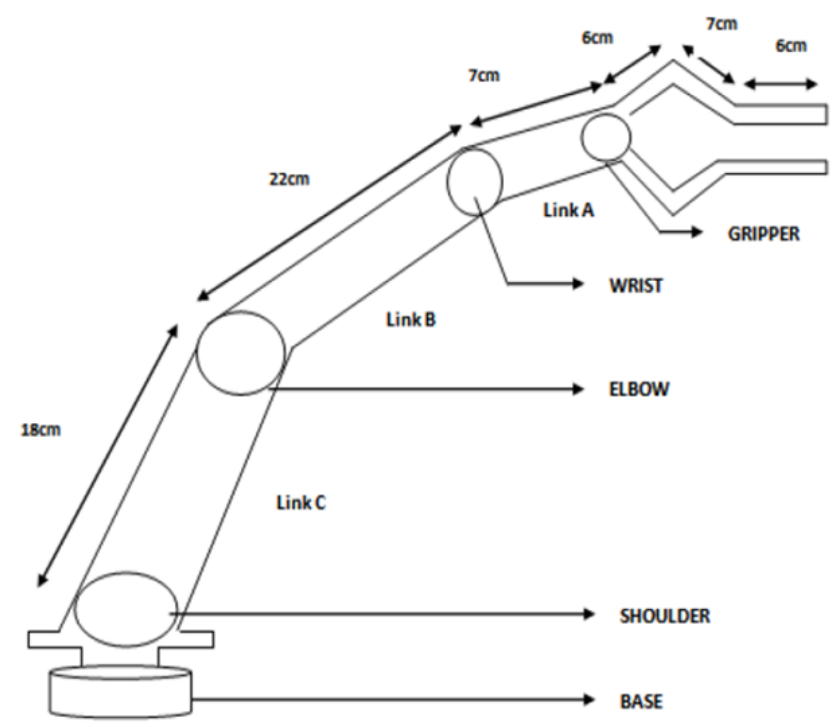

Fig. 2 Design of the Robotic Manipulator (arm)

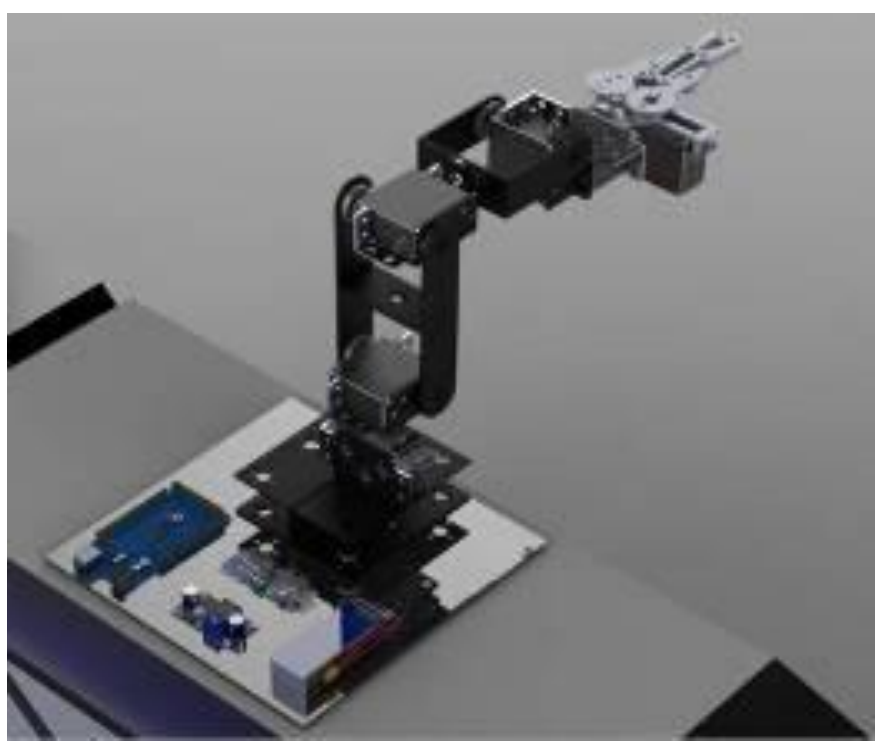

Fig. 3 Mechanical Design

Fig. 4 represents the flowchart of the pick and place robot. As the system starts, image processing using OpenCV with Haar Cascade Method is applied. Then, camera will sort out the products. If the product is yellow and large, the product will be placed by robot in $90^{\circ}$ left side and if the product is small, the product will be placed in $45^{\circ}$ left side. Moreover, if the color of product is black and large, then the robot will place the product in $90^{\circ}$ right direction and if it is a small product, then the product will be placed in $45^{\circ}$ right direction. All process is done with net framework, Arduino Nano and OpenCV shows the resultant output in laptop. 


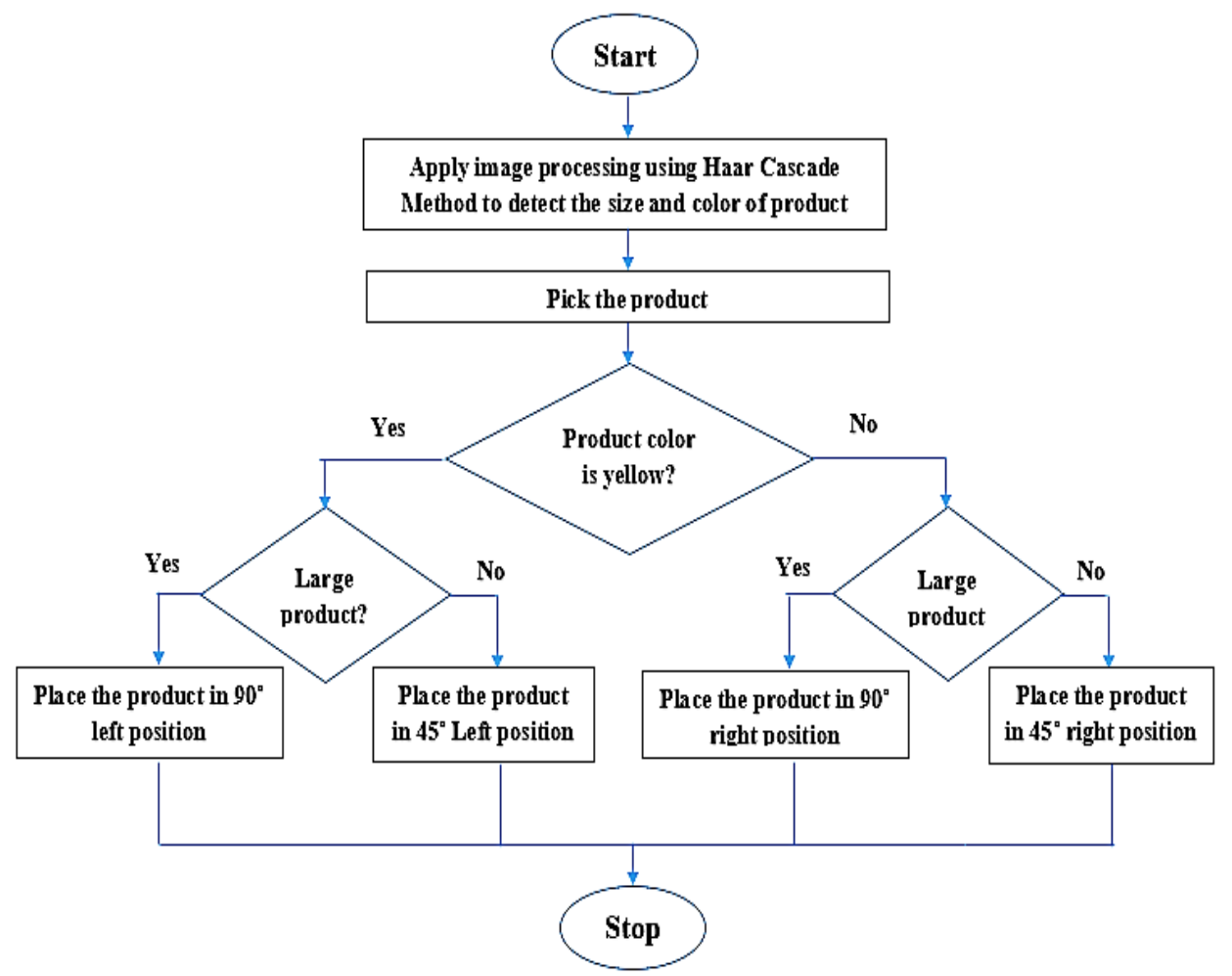

Fig. 4 Flowchart

\section{Results Analysis}

In this color and height sorting pick and place robot, Arduino Nano is connected with dc motor, servo motors and PC. Experimental setup is given in Fig. 5. The connection between Arduino to $\mathrm{I} / \mathrm{O}$ with robot and camera feedback is done by the signal flow of block diagram and it's shown in Fig. 1.

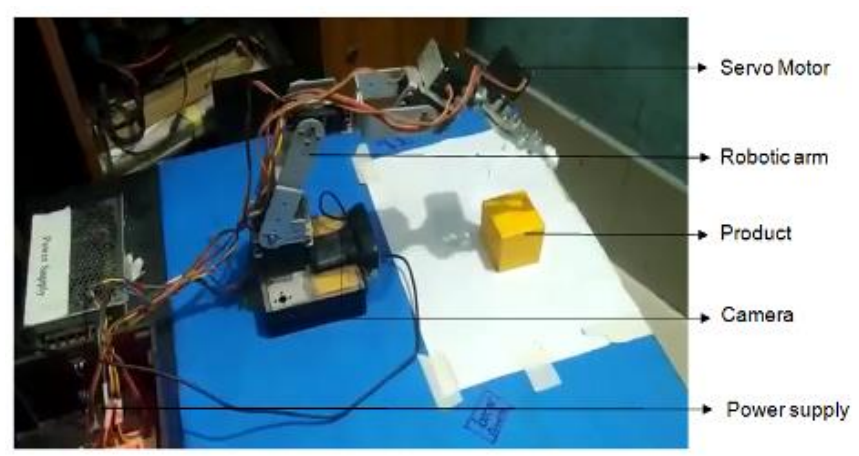

Fig. 5 Hardware Setup

Table 2 shows D-H parameters 0 of a 6 DOF Manipulator for this pick and place robot.

Table 2 D-H Parameters of a 6 DOF Manipulator

\begin{tabular}{ccccc}
\hline Joint $_{\mathrm{i}}$ & $\alpha_{\mathrm{i}}$ & $\mathrm{a}_{\mathrm{i}}$ & $\mathrm{d}_{\mathrm{i}}$ & $\theta_{\mathrm{i}}$ \\
\hline 1 & 0 & $\mathrm{a}_{1}$ & $\mathrm{~d}_{1}$ & $\theta_{1}$ \\
2 & 0 & $\mathrm{a}_{2}$ & 0 & $\theta_{2}$ \\
3 & $-\pi / 2$ & $\mathrm{a}_{3}$ & 0 & $\theta_{3}$ \\
4 & $-\pi / 2$ & $\mathrm{a}_{4}$ & 0 & $\theta_{4}$ \\
5 & 0 & 0 & 0 & $\theta_{5}$ \\
6 & 0 & 0 & $\mathrm{~d}_{6}$ & $\theta_{6}$ \\
\hline
\end{tabular}

$$
{ }^{0} A_{1}=\left[\begin{array}{cccc}
c \theta_{1} & -s \theta_{1} & 0 & a_{1} c \theta_{1} \\
s \theta_{1} & c \theta_{1} & 0 & a_{1} s \theta_{1} \\
0 & 0 & 1 & d_{1} \\
0 & 0 & 0 & 1
\end{array}\right]
$$

$$
{ }^{3} A_{4}=\left[\begin{array}{cccc}
c \theta_{4} & 0 & -s \theta_{4} & a_{4} c \theta_{4} \\
s \theta_{4} & 0 & c \theta_{4} & a_{4} s \theta_{4} \\
0 & -1 & 0 & 0 \\
0 & 0 & 0 & 1
\end{array}\right]
$$


Multiplying equations yields:

${ }^{o} A_{6}={ }^{o} A_{1}{ }^{* 1} A_{2}{ }^{* 2} A_{3}{ }^{*} A_{4}{ }^{* 4} A_{5}{ }^{* 5} A_{6}$

${ }^{o} A_{6}=\left[\begin{array}{cccc}U x & V x & W x & Q x \\ U y & V y & W y & Q y \\ U z & V z & W z & Q z \\ 0 & 0 & 0 & 1\end{array}\right]$

$U X=C \theta_{123456}-C \theta_{3456} S \theta_{12}-C \theta_{1456} S \theta_{13}-$

$C \theta_{2456} S \theta_{13}+C \theta_{126} S \theta_{35}-$

$S \theta_{1235}+C \theta_{136} S \theta_{25}+C \theta_{236} S \theta_{15}-C \theta_{1234} S \theta_{56}$

$+C \theta_{34} S \theta_{1256}+C \theta_{14} S \theta_{1356}+C \theta_{24} S \theta_{1356}+C \theta_{125} S \theta_{36}-$

$S \theta_{1236}+C \theta_{135} S \theta_{26}+C \theta_{235} S \theta_{16}$

$U y=C \theta_{23456} S \theta_{1}+C \theta_{13456} S \theta_{4-}$

$S \theta_{123} C \theta_{456}+C \theta_{12456} S \theta_{3}+C \theta_{26} S \theta_{135}+C \theta_{16} S \theta_{235}+S \theta$

${ }_{125} C \theta_{36}-C \theta_{1236}-C \theta_{234} S \theta_{156}-C \theta_{134} S \theta_{256}-S \theta_{12356} C \theta_{4}$

$+C \theta_{124} S \theta_{356}+C \theta_{25} S \theta_{136}+C \theta_{15} S \theta_{236}+S \theta_{126} C \theta_{35}$

$C \theta_{1235}$

$U Z=-C \theta_{56} S \theta_{4}-S \theta_{456}$

$V X=-C \theta_{12345} S \theta_{6}+C \theta_{345} S \theta_{126}+C \theta_{145} S \theta_{136}$ $+C \theta_{245} S \theta_{136}-C \theta_{24} S \theta_{356}+S \theta_{12356}-C \theta_{13} S \theta_{256}-$ $C \theta_{23} S \theta_{156}-C \theta_{12346} S \theta_{5}+C \theta_{346} S \theta_{125}+C \theta_{146} S \theta_{135}$ $+C \theta_{246} S \theta_{135}+C \theta_{1256} S \theta_{3}-S \theta_{123}+C \theta_{1356} S \theta_{2}$ $+C \theta_{2356} S \theta_{1}$

$V y=-C \theta_{2345} S \theta_{16}-C \theta_{1345} S \theta_{26}+S \theta_{1236} C \theta_{45}-C \theta_{1245}$

$S \theta_{36}-C \theta_{2} S \theta_{1356}-C \theta_{1} S \theta_{2356}-S \theta_{1256} C \theta_{3}+C \theta_{123}-$

$C \theta_{2346} S \theta_{15}-C \theta_{1346} S \theta_{25}-S \theta_{1235} C \theta_{46}+C \theta_{1246} S \theta_{35}$

$+C \theta_{256} S \theta_{13}+C \theta_{156} S \theta_{23}+S \theta_{12} C \theta_{356}-C \theta_{12356}$

$V z=C \theta_{5} S \theta_{46}-S \theta_{456}$

$W_{X}=-C \theta_{123} S \theta_{4}+C \theta_{3} S \theta_{24}+C \theta_{1} S \theta_{134}+C \theta_{2} S \theta_{134}$

$W y=-C \theta_{23} S \theta_{14}-C \theta_{13} S \theta_{24}+S \theta_{1234}-C \theta_{12} S \theta_{34}$

$W z=-C \theta_{4}$

$Q_{X}=-d_{6} C \theta_{123} S \theta_{4}+d_{6} C \theta_{3} S \theta_{124}+d_{6} C \theta_{1} S \theta_{134}+$ $d_{6} C \theta_{2} S \theta_{134}+a_{4} C \theta_{1234}-a_{4} C \theta_{34} S \theta_{12}-C \theta_{14} S \theta_{13}-$ $C \theta_{24} S \theta_{13}+a_{3} C \theta_{123}-a_{3} C \theta_{3} S \theta_{12}-a_{3} C \theta_{1} S \theta_{23}-a_{3} C \theta_{2} S \theta_{13}$ $+a_{2} C \theta_{12}-a_{2} S \theta_{12}+a_{1} C \theta_{1}$

$Q y=-d_{6} C \theta_{23} S \theta_{14}-d_{6} C \theta_{13} S \theta_{24}+d_{6} S \theta_{1234}-d_{6} C \theta_{12}$

$S \theta_{34}+a_{4} C \theta_{234} S \theta_{1}+a_{4} C \theta_{134} S \theta_{2}-a_{4} C \theta_{4} S \theta_{123}$

$+a_{4} C \theta_{124} S \theta_{3}+a_{3} C \theta_{23} S \theta_{1}+a_{3} C \theta_{13} S \theta_{2}-a_{3} S \theta_{123}$

$+a_{3} C \theta_{12} S \theta_{3}+a_{2} C \theta_{2} S \theta_{1}+a_{2} C \theta_{1} S \theta_{2}+a_{1} S \theta_{1}$

$Q Z=-d_{6} C \theta_{4}-a_{4} S \theta_{4}+d_{1}$

When the black and large object is detected, it shows "Black object and large box detected" in OpenCV which is illustrated in Fig. 6. Furthermore, when yellow and large object is detected, then it shows "Yellow object and large box is detected" in OpenCV which is illustrated in Fig. 7. Furthermore, OpenCV shows "Black object and small box detected" when camera detects black and small object, which is shown in Fig. 8.

To perform this task with more objects in dataset for qualitative results, it uses yellow with small object, red with large and small objects, blue with large and small objects and, at last green with large and yellow objects. So, our dataset contains all of these objects and we can detect all the objects successfully.

With the help of confusion matrix, we get the precision and recall rates for our work, which shows that, our work is quite effective to work with. below.

Confusion matrix with precision and recall rates are shown

Confusion matrix $=$

$$
\begin{gathered}
{[[96,1,0,3,0,0,0,0,0,0],} \\
{[1,98,0,0,0,0,1,0,0,0],} \\
{[0,0,100,0,0,0,0,0,0,0],} \\
{[1,0,0,95,3,1,0,0,0,0],} \\
{[0,0,3,0,95,0,0,0,0,2],} \\
{[0,2,0,0,1,97,0,0,0,0],} \\
{[0,0,0,0,0,0,100,0,0,0],} \\
{[0,2,1,0,0,1,0,94,2,0],} \\
{[1,0,0,0,0,1,0,0,97,1],} \\
[0,0,0,0,0,0,0,0,0,100]])
\end{gathered}
$$

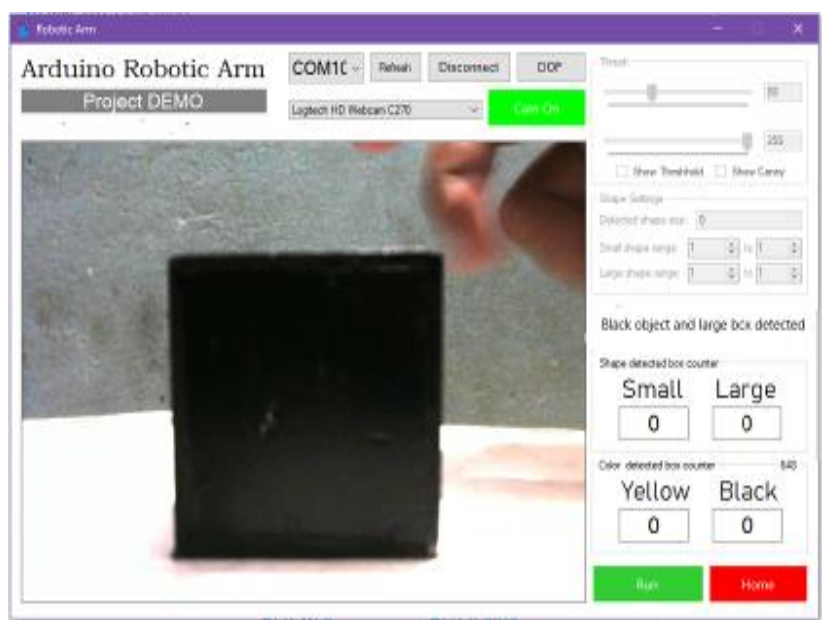

Fig. 6 OpenCV Detecting the Black and Large Object

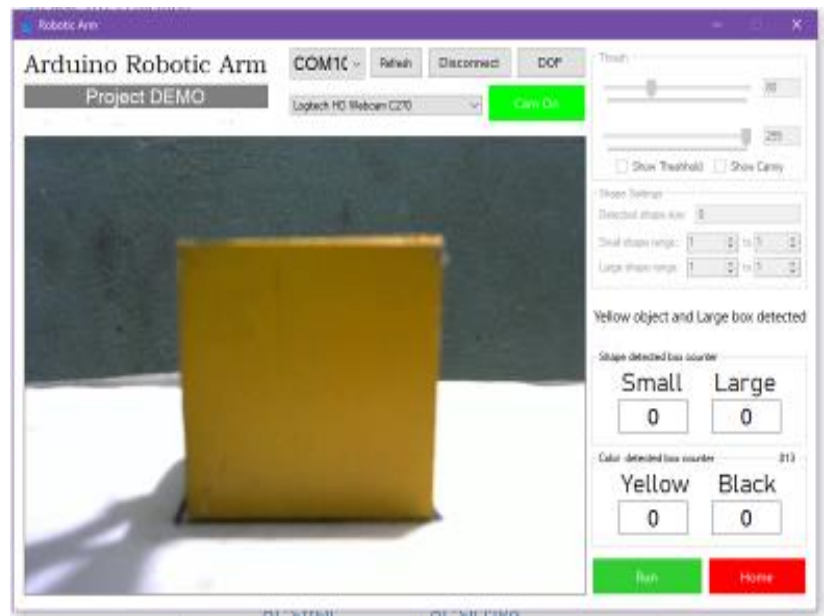

Fig. 7 OpenCV Detecting the Yellow and Large Object

Precision and recall rates for the system is shown below. Label precision recall

$\begin{array}{lll}0 & 0.970 & 0.960 \\ 1 & 0.951 & 0.980 \\ 2 & 0.962 & 1.000 \\ 3 & 0.969 & 0.950 \\ 4 & 0.960 & 0.950 \\ 5 & 0.970 & 0.970 \\ 6 & 0.990 & 1.000 \\ 7 & 1.000 & 0.940 \\ 8 & 0.980 & 0.970 \\ 9 & 0.971 & 1.000\end{array}$




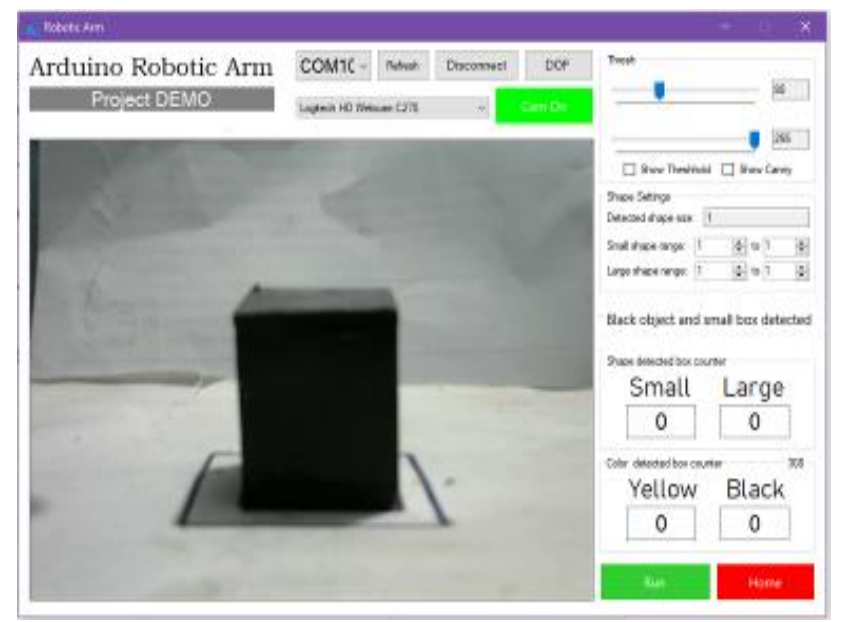

Fig. 8 OpenCV Detecting the Black and Small Object

This work is done with white background and level of illumination should be brightest as possible. When we did our qualitative task, for yellow object, two times it's failed to detect because of lower brightness.

DC servo motor has low power requirement and the light weight makes it suitable for this design. The torque is fully balanced by the inertia of the electric motors and the speed is significantly reduced by gear sets attached to the electric motors. In this aspect, several calculations were performed in order to attain the required servo mechanism for meeting the specification required for this work. Fig. 9 shows the torque calculation for each servo motor.

Numerous calculations were done in order to attain the required servo mechanism that will meet the specification on this work.

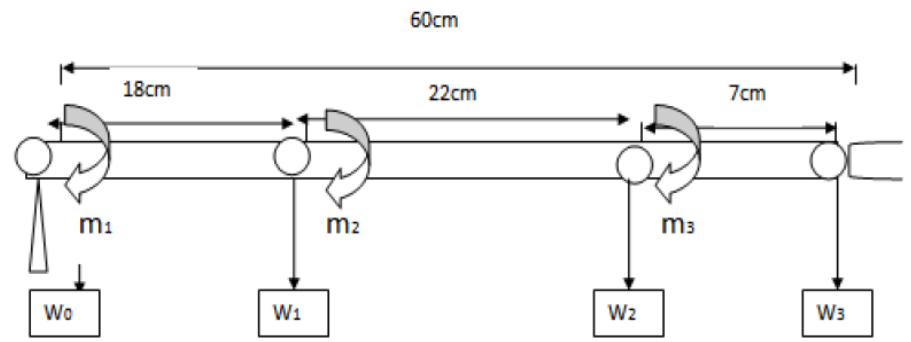

Fig. 9 Diagram Showing Torque Calculation on Each Servo

Assuming that the weight of the material is negligible since it light compares to the servo specification. $\mathrm{W}_{0}=56 \mathrm{~g}, \mathrm{~W}_{1}=56 \mathrm{~g}$, $\mathrm{W}_{2}=56 \mathrm{~g}, \mathrm{~W}_{3}=36 \mathrm{~g}$, base weight $=56+56+56+36=204 \mathrm{~g}$, (weights include gravitational force $[\mathrm{f}=\mathrm{mg}])$, base length $=10 \mathrm{~cm}$.

From Newton-Euler recursive formulation 0, we find that, for torque,

Outward iterations: i: $0 \rightarrow 5$

$$
\begin{aligned}
& { }^{i+1} \omega_{i+1}={ }_{i}^{i+1} R^{i} \omega_{i}+\dot{\theta}_{i+1}{ }^{i+1} \widehat{Z}_{i+1} \\
& { }^{i+1} \dot{\omega}_{i+1}={ }^{i+1} \widehat{ }_{i}{ }^{i} \omega_{i}+{ }^{i+1}{ }_{i}{ }^{i} \omega_{i} X \dot{\theta}_{i+1}{ }^{i+1} \widehat{Z}_{i+1}+\ddot{\theta}_{i+1} \\
& { }^{i+1} \dot{v}_{i+1}={ }^{i+1} R\left({ }_{i} \omega_{i} X^{i} P_{i+1}+{ }^{i} \omega_{i} X\left({ }^{i} \omega_{i} X^{i} P_{i+1}\right)+{ }^{i} \dot{v}_{I}\right. \\
& { }^{i+1} \dot{v} c_{i+1}={ }^{i+1} \dot{\omega}_{i+1} X^{i} P C_{i+1}+{ }^{i+1} \omega_{i+1} X\left({ }^{i+1} \omega_{i+1} X\right. \\
& \left.{ }^{i} P C_{i+1}\right)+{ }^{i+1} \dot{v}_{i+1} \\
& { }^{i+1} F_{i+1}=m_{i+1}{ }^{I+1} \dot{v} c_{i+1} \\
& { }^{i+1} N_{i+1}={ }^{c i+1} I_{i+1}{ }^{i+1} \dot{\omega}_{i+1}+{ }^{i+1} \omega_{i+1} X^{c i+1} I_{i+1}{ }^{i+1} \omega_{i+1}
\end{aligned}
$$

Inline iterations: i: $6 \rightarrow 1$

$$
\begin{aligned}
& { }^{i} f_{i}={ }_{i+1}^{i} R{ }^{i+1} f_{i+1}+{ }^{i} F_{i} \\
& { }^{i} n_{i}={ }^{i} N_{i}+{ }_{i+1}^{i} R^{i+1} n_{i+1}+{ }^{i} P C_{i} X^{i} F_{i}+{ }^{i} P_{i+1} X_{i+1}{ }^{i} R{ }^{i+1} f_{i+1} \\
& \gamma_{i}={ }^{i} n_{i}^{T}{ }^{i} \hat{Z}_{i}
\end{aligned}
$$

Finally, for translational joint,

$$
\varphi_{i=} f_{i}\left(R_{i-1}^{i}\right)^{T} Z
$$

For angular joint,

$$
\varphi_{i=n_{i}}\left(R_{i-1}^{i}\right)^{T} Z
$$

After calculating all of this, for $6 \mathrm{DOF}$ robotic arm, for torque, we find,

$$
\begin{array}{cccc}
L & \dot{p} I x x & -q r I y y & +q r I z z \\
M= & \dot{q} I x x & +p r I y y & -p r I z z \\
N & \dot{r} I x x & -p q I y y & +p q I z z
\end{array}
$$

And, for force,

$$
\begin{array}{lll}
F x & u & +(q w-r v) \\
F y=v & +(r u-p w) \\
F z & w & +(p v-q u)
\end{array}
$$

Where,

$\mathrm{I}, \mathrm{j}, \mathrm{k}=$ body center coordinate in $\mathrm{x}, \mathrm{y}$ and $\mathrm{z}$ directions $\mathrm{u}, \mathrm{v}, \mathrm{w}=$ linear velocities in $\mathrm{x}, \mathrm{y}$ and $\mathrm{z}$ directions $\mathrm{p}, \mathrm{q}, \mathrm{r}=$ angular velocity in $\mathrm{x}, \mathrm{y}$ and $\mathrm{z}$ directions Ixx, Iyy, Izz $=$ Moment of inertia in $\mathrm{x}, \mathrm{y}$ and $\mathrm{z}$ directions

Since moment of inertia of $\mathrm{z}$-axis is zero, so torque,

$$
\begin{array}{crll}
L & \dot{p} I x x & -q r I y y & 0 \\
M= & \dot{q} I x x & +p r I y y & 0 \\
N & \dot{r} I x x & -p q I y y & 0
\end{array}
$$

With the help of parallel axis theorem we find,

$$
\begin{aligned}
& I_{x x}=I_{x x 1}+M d_{x}^{2} \\
& I_{y y}=I_{y y 1}+M d_{y}^{2}
\end{aligned}
$$

Because the axis of rotation is on the edge, so

$$
\begin{aligned}
& I_{x x 1}=M b^{3} / 3 \\
& I_{y y 1}=M h^{3} / 3
\end{aligned}
$$

After calculating, we find

$$
\begin{aligned}
& \mathrm{I}_{\mathrm{xx}}=0.00413 \mathrm{~kg}-\mathrm{cm}^{2} \\
& \mathrm{I}_{\mathrm{yy}}=38 \mathrm{~km}-\mathrm{cm}^{2}
\end{aligned}
$$

For angular velocity,

$$
{ }^{6} \omega_{6}=\begin{gathered}
0 \\
0 \\
\dot{\theta} 1+\dot{\theta} 2+\dot{\theta} 3+\dot{\theta} 4+\dot{\theta} 5+\dot{\theta} 6
\end{gathered}
$$

So, angular velocity, $\mathrm{p}$ and $\mathrm{q}=0$.

$$
r=\dot{\theta} 1+\dot{\theta} 2+\dot{\theta} 3+\dot{\theta} 4+\dot{\theta} 5+\dot{\theta} 6=18.18 \mathrm{rad} / \mathrm{s}
$$

So, torque,

$$
\begin{aligned}
& \begin{array}{llll}
L & 0 & 0 & 0
\end{array} \\
& \begin{array}{l}
M= \\
N
\end{array} \begin{array}{ccc}
0 & 0 & 0 \\
0.00413(\dot{\theta} 1+\dot{\theta} 2+\dot{\theta} 3+\dot{\theta} 4+\dot{\theta} 5+\dot{\theta} 6) & 0 & 0
\end{array}
\end{aligned}
$$

So, $\mathrm{N}_{1}=0.076818 \mathrm{~kg}-\mathrm{cm}$ 
Actual torque of the shoulder servo $=13[\mathrm{~kg}-\mathrm{cm}]($ From datasheet)

Excess torque $=$ Actual servo torque - Calculated torque

Therefore excess available torque at the shoulder $=13-$ $0.076818=12.92[\mathrm{~kg}-\mathrm{cm}]$

From the design analysis the maximum load the robotic manipulator can lift successfully is determine by the base servo. From the above analysis the excess torque of the base is 12.92 $[\mathrm{kg}-\mathrm{cm}]$. Hence, the calculated maximum load is $12.92[\mathrm{~kg}]$. The actual load will be less than the calculated value because the weight of the material used in constructing the arm was light and was not taken into consideration.

For linear velocity, $w=0$. We also find $\mathrm{p}, \mathrm{q}=0$. After calculating, we get, $\mathrm{u}=2 \mathrm{~m} / \mathrm{s}, \mathrm{v} \approx 0$.

So, force,

$$
\begin{array}{lccc}
F x & u & 0 & 2 \\
F y= & 0 & +18.18 u & =36.6 \\
F z & 0 & 0 & 0
\end{array}
$$

So, maximum force can be given as $2 \mathrm{~N}$ and $36.36 \mathrm{~N}$ for $\mathrm{x}$ and y direction respectively.

This system is quite effective and efficiency of the system is almost $99 \%$.

\section{Conclusion and Future Work}

Since robots are used in flexible or even fixed automation systems, there are two reasons for selecting a robot to operate in a production line. The reasons include reducing labor costs and performing work that is tedious, unpleasant and hazardous for human beings. However, introducing too many robots for picking and placing is quite expensive and risky as well. Therefore, this work has introduced where only one robotic arm is used which is competent in picking and placing objects based on color and height. The whole process is regulated with Arduino Nano. The applications of this work are suited for industry, where product with different color and height can be sorted out.

The work can further be improved in the near future by introducing the conveyor belt, in which, the objects or products can come to a certain place and sorting robot can be used for picking the products. The conveyor belts can also be used for placing the objects or products according to their colors and sizes.

\section{References}

Dhayalini, K. and Mukesh, R., 2018, January. Deterioration $\&$ non-deterioration wastes separation using pick \& place robot. In 2018 2nd International Conference on Inventive Systems and Control (ICISC) (pp. 96-99). IEEE.

$\mathrm{Hu}$, C.Y., Chen, C.R., Tseng, C.H., Yudha, A.P. and Kuo, C.H., 2016, March. Visual servoing spanner picking and placement with a SCARA manipulator. In 2016 IEEE
International Conference on Industrial Technology (ICIT) (pp. 1632-1637). IEEE.

Gecks, T. and Henrich, D., 2005, August. Human-robot cooperation: safe pick-and-place operations. In ROMAN 2005. IEEE International Workshop on Robot and Human Interactive Communication, 2005. (pp. 549-554). IEEE.

Kato, G., Onchi, D. and Abarca, M., 2013. Low cost flexible robot manipulator for pick and place tasks. In $201310^{\text {th }}$ International Conference on Ubiquitous Robots and Ambient Intelligence (URAI) (pp. 677-680). IEEE.

Andhare, P. and Rawat, S., 2016, August. Pick and place industrial robot controller with computer vision. In 2016 International Conference on Computing Communication Control and automation (ICCUBEA) (pp. 1-4). IEEE.

Li, W., Xiao, Y., Bi, S. and Du, G., 2013, September. Automatic elliptical trajectory planning algorithm for pick and place operation. In 2013 International Conference on Advanced Mechatronic Systems (pp. 36-39). IEEE.

Lin, H.I., Chen, Y.Y. and Chen, Y.Y., 2015, May. Robot vision to recognize both object and rotation for robot pickand-place operation. In 2015 International Conference on Advanced Robotics and Intelligent Systems (ARIS) (pp. 1-6). IEEE.

Lukač, D., 2018, May. Simulation of a pick-and-place cube robot by means of the simulation software KUKA Sim Pro. In 2018 41st International Convention on Information and Communication Technology, Electronics and Microelectronics (MIPRO) (pp. 0846-0849). IEEE.

Rahman, M.J., Das, D.P., Islam, O. and Zaman, H.U., 2018, February. A Novel Design of a Robotic Object Sorter Based on Color Differences using Image Processing Techniques. In 2018 International Conference on Computer, Communication, Chemical, Material and Electronic Engineering (IC4ME2) (pp. 1-4). IEEE.

Viola, P. and Jones, M., 2001, December. Rapid object detection using a boosted cascade of simple features. In 2001 IEEE computer society conference on computer vision and pattern recognition $(C V P R)$ (Vol. 1, pp. I-I). IEEE.

Roshni, N. and Kumar, T.S., 2017, March. Pick and place robot using the centre of gravity value of the moving object. In 2017 IEEE International Conference on Intelligent Techniques in Control, Optimization and Signal Processing (INCOS) (pp. 1-5). IEEE.

Rahmad, C., Asmara, R.A., Putra, D.R.H., Dharma, I., Darmono, H. and Muhiqqin, I., 2020, January. Comparison of Viola-Jones Haar Cascade Classifier and Histogram of Oriented Gradients (HOG) for face detection. In IOP Conference Series: Materials Science and Engineering (Vol. 732, No. 1, p. 012038). IOP Publishing.

Craig, J.J., 2009. Introduction to robotics: mechanics and control, 3/E. Pearson Education India. 